\title{
Relación entre la atención y el rendimiento escolar en niños y adolescentes
}

\section{Relation Relation Between Attention and Achievement at School in Children and Adolescents}

\author{
Santiago Resett \\ Universidad Argentina de la Empresa-CONICET, \\ Buenos Aires, Argentina
}

\begin{abstract}
Resumen
El rendimiento escolar en niños y adolescentes es una tarea esencial del desarrollo con implicancias psicosociales contemporáneas como a largo plazo. Un importante constructo neuropsicológico es la atención, la cual está en la base de cualquier aprendizaje. Objetivos: la presente investigación trató de determinar si el desempeño atencional de niños y adolescentes era un predictor de las calificaciones escolares. Métodos: se constituyó una muestra de 82 niños de tres cursos en una escuela primaria privada en Paraná, Entre Ríos, Argentina. Además, se constituyó una muestra de 73 adolescentes de dos cursos en una escuela secundaria privada en Paraná, Entre Ríos, Argentina. Los participantes completaron el d2 Test de Atención Brickenkamp y se solicitaron las calificaciones de todas las materias escolares. Los datos se procesaron cuantitativamente con el programa SPSS 23. Resultados: tanto en el caso de los niños como los adolescentes, las calificaciones más bajas se hallaban en Matemática y Lengua. El total de respuestas y de aciertos eran predictores significativos de las calificaciones en Matemática, Lengua y Ciencias Básicas en niños. En el caso de los adolescentes, también, el total de respuestas y aciertos predecían las notas de Matemática, Lengua, Inglés y Biología, aunque en todos los casos el tamaño del efecto era pequeño. Se observó un desempeño atencional más elevado en los adolescentes en comparación con los niños. Discusión: los hallazgos del presente estudio sugieren que el desempeño atencional es un predictor significativo del rendimiento escolar de los niños y los adolescentes.
\end{abstract}

Palabras clave: neurociencias, atención, rendimiento, escuela, niños, adolescentes

Santiago Resett, Universidad Argentina de la Empresa-CONICET, Buenos Aires, Argentina.

La correspondencia en relación con este artículo se dirige a Santiago Resett, Universidad Argentina de la Empresa-CONICET, Buenos Aires, Argentina. Dirección electrónica: resettsantiago@gmail.com 


\begin{abstract}
School performance in children and adolescents is an important development task with notable psychosocial correlates, both contemporary and long term. An important neuropsychological construct is attention, which is the basis of any learning. Objectives: the present investigation was oriented to determine if attention performance in children and adolescents was a predictor of school grades. Method: a sample of 82 children from three grades was selected at a private primary school in Paraná, Entre Ríos, Argentina. In addition, a sample of 73 adolescents from two grades was selected at a private secondary school in Paraná, Entre Ríos, Argentina. Participants completed the Brickenkamp d2 Attention Questionnaire and grades for all school subjects were obtained. Results: in the cases of both children and adolescents, their lowest grades were in Mathematics and Language. The total of responses and correct answers were significant predictors of the grades in Mathematics, Language and Basic Sciences in children. In the case of adolescents, total responses and accuracy predicted the Math, Language, English and Biology grades, although in all cases the effect size was small. Higher attentional performance was observed in adolescents, compared to children. Discussion: the findings of the present study suggest that attentional performance is a significant predictor of school performance in children and adolescents.
\end{abstract}

Keywords: Neurosciences, Attention, Achievement, School, Children, Adolescents.

En las últimas décadas, las neurociencias tuvieron un desarrollo notable. En este sentido, la década de 1990 se denominó la década "del cerebro" (Manes, 2014; Rodríguez, 2004). Los orígenes de dicha revolución se remontan a 1988 en los Estados Unidos, país donde se lanza un ambicioso proyecto titulado "la década del cerebro". Dicho proyecto proponía no solo conocer el sistema nervioso, sino también prevenir las enfermedades neurológicas y mejorar el desempeño de las funciones cognitivas. De este modo, en el último tiempo, el conocimiento del funcionamiento y la estructura del sistema nervioso han sido sumamente exhaustivo, principalmente, gracias a las técnicas de neuroimágenes tales como la tomografía computada o la tomografía por emisión de positrones (Portellano, 2005; Rodríguez, 2004).

Un importante constructo, que ha generado una vasta investigación dentro de las neurociencias y de la "década del cerebro", fue la atención. La atención se define como la función responsable de la activación y el funcionamiento de los procesos de selección, distribución y mantenimiento de la actividad mental (Portellano, 2005; Ríos-Lago et al., 2007). Hace más de un siglo, William James (1890) la había definido proféticamente como la capacidad de tomar o considerar -de una forma clara y vivida- los diferentes objetos o estímulos que existen a nuestro alrededor.

Como muchas funciones mentales, la atención es un proceso complejo y compuesto por distintos mecanismos - con diferentes sustratos neuronales- (Bryck \& Fisher, 2011; Gogtay et al., 2004), lo cual dio lugar a distintas clasificaciones (Brefczynski-Lewis et al., 2007; Jiménez et al., 2012; Portellano, 2005). Así, es un proceso subyacente para la mayoría de las funciones psicológicas y mentales. Por ejemplo, la atención está en la base del funcionamiento cognitivo, tanto por la relevancia de su función en sí, por ser la mediadora de otros procesos cognitivos, como la memoria o el aprendizaje (Chun \& TurkBrowne, 2007; Rumbaugh \& Washburn, 1995). Por lo tanto, constituye uno de los procesos más importantes de la cognición humana, porque participa en el resto de las funciones psicológicas (Portellano, 2005) o en distintas tareas como el aprendizaje escolar. Esto se debe a que entre sus principales funciones 
RELACIÓN ENTRE LA ATENCIÓN Y EL RENDIMIENTO ESCOLAR EN NIÑOS Y ADOLESCENTES

se encuentra la de seleccionar los estímulos relevantes del ambiente a expensas de otros elementos menos relevantes (Pashler, 1998). Posner -uno de los investigadores más relevantes en este constructo- y sus colegas desarrollaron una detallada taxonomía empírica de la atención al postular que no es una función de un área cerebral específica ni tampoco de todo el cerebro (Fan et al., 2002; Fan et al., 2005; Posner \& Dehaene, 1994; Posner \& Peterson, 1990; Rothbart et al., 2011). Al contrario, puede ser entendida como tres redes funcionales y anatómicamente definidas -las cuales interactúan entre sí permanentementeaunque cumplan tareas específicas (Petersen \& Posner, 2012). De acuerdo con las concepciones recientes y con dichos autores, estas redes de atención son las siguientes: alerta, orientación y atención ejecutiva (Petersen \& Posner, 2012; Rothbart et al., 2011). La red de alerta se encarga de la capacidad de respuesta del organismo frente a la estimulación sensorial; en otras palabras, regula y mantiene la activación y la vigilia. La red de orientación selecciona un subconjunto de información o estímulo para su procesamiento; por lo tanto, esta red entra en juego cuando los recursos atencionales se orientan hacia un estímulo de interés. Se han propuesto muchos factores para explicar los efectos beneficiosos de la orientación atencional tales como los refuerzos neuronales (Luck et al., 1996; Mangun \& Hillyard, 1991), la supresión de un distractor (Reynolds et al., 2000; Slotnick et al., 2003) y/o la reducción del ruido (Dosher \& Lu, 2000). Finalmente, la red de atención ejecutiva se encarga de las representaciones post-sensoriales y ayuda a regular nuestra conducta - ante un sistema central de capacidad limitada- al inhibir los distractores y monitorear la conducta para una mejor adaptación a nuestro ambiente (Petersen \& Posner, 2012). Con respecto a las diferencias entre niños y adolescentes en la atención, está comprobado que los adolescentes presentan un desempeño atencional más elevado: mejor focalización, mayor capacidad de inhibición, entre otros (Arnett, 2012).

Debido a la gran relevancia de dicha variable, el desempeño atencional se asocia con numerosas patologías físicas y trastornos mentales como las demencias -el trastorno de Alzheimer, por ejemplo-, la epilepsia, los trastornos del desarrollo, los trastornos emocionales, como el trastorno depresivo mayor o la disforia y el déficit de atención (Biederman et al., 2004). El caso más conocido de una patología mental asociada con dificultades atencionales es -sin dudas- el déficit de atención con hiperactividad (ADHD por su acrónimo en el idioma inglés) y sin hiperactividad (ADD). Este es un trastorno de desarrollo neuronal con síntomas de hiperactividad, impulsividad y dificultades en el rendimiento atencional (Biederman et al., 2004), causado por problemas en determinados neurotrasmisores como la dopamina y la norepinefrina (Gottlieb, 2007). El Manual Diagnóstico y Estadístico de los Trastornos Mentales en su quinta edición ([DSM-V] Asociación Americana de Psiquiatría, 2013) señala que, para ser diagnosticado con dicho trastorno, el sujeto debe presentar -al menos- cinco síntomas relacionados con dificultades atencionales. Se estima que entre un $5 \%$ y un $7 \%$ de niños de edad escolar presentan dicho trastorno (Thomas et al., 2015). Este trastorno se asocia a un sinnúmero de problemas de ajuste psicosocial como mayores niveles de agresividad, mayor estrés en los cuidadores del niño o un deterioro del rendimiento escolar (Barkley, 1997; LeFever et al., 2002).

Debido al incremento de los niveles de escolaridad alcanzado por los niños y adolescentes a lo largo de todo el siglo XX en las naciones industrializadas y posindustrializadas (Arnett, 2012), el rendimiento escolar se ha vuelto una variable de gran relevancia psicosocial (Steinberg, 1997). Genéricamente, esta variable se operacionaliza como el grado o nivel de logro en conocimientos, saberes y/o capacidades 
establecidos en los programas oficiales de estudio (Caso-Niebla \& Hernández-Guzmán, 2007; Steinberg, 1997). En cambio, otra operacionalización apunta a la evaluación de los procesos educativos formales como el de las instituciones escolares o educativas (Valle-Arias et al., 2000). Aunque no existe un consenso en su definición, la mayoría de los investigadores lo define como una medida estimativa de las capacidades, las aptitudes o los conocimientos que un alumno aprendió como resultado de un proceso de instrucción formal (Rumberger \& Palardy, 2005).

Está comprobado que el rendimiento académico o escolar corresponde a una de las tareas del desarrollo psicosocialmente más importante para niños y adolescentes, porque se asocia con un mejor ajuste psicosocial, como menores problemas emocionales y de conducta, no solo concurrentes, sino también a largo plazo (Arnett, 2012; Steinberg, 2019). También, es un poderoso factor predictivo de un mejor ajuste psicosocial en la adultez como un mejor ingreso económico, menores problemas de salud, mejores ocupaciones y menores problemas emocionales y de conducta (Steinberg, 2019). La importancia psicosocial de dicho constructo para niños y adolescentes es que -a diferencia de otros aspectos psicosociales de su vida- este se evalúa de acuerdo con parámetros objetivos que permiten la comparación del rendimiento del niño o adolescente con sus pares (Steinberg, 2019). Por lo tanto, los logros educativos de un sujeto determinan la futura inserción en el mercado de trabajo y en la educación universitaria, entre otros aspectos (Arnett, 2012; Steinberg, 2019).

A pesar de la relevancia del rendimiento escolar, su definición y medición no es sencilla, debido a que diversas operacionalizaciones se han brindado al respecto (Rumberger \& Palardy, 2005). Así, para evaluar el rendimiento escolar de un sujeto, se pueden tomar los puntajes obtenidos por los estudiantes -las notas o calificaciones-, las tasas de deserción o abandono escolar, el retraso escolar o la repitencia, la participación escolar, el ausentismo y las actitudes hacia las escuela, entre otros (Rumberger \& Palardy, 2005; Steinberg, 2019).

Sin embargo, la forma más frecuente de medirlo -aunque no la única- es mediante la evaluación de las destrezas o las capacidades cognitivas que obtienen los alumnos en tests estandarizados durante un período lectivo o curso (Galicia-Moyeda et al., 2013; Pizarro et al., 1987); esto es, mediante las calificaciones escolares obtenidas por los alumnos. No obstante, esta forma frecuente de evaluarlo tiene sus falencias o debilidades, porque estos indicadores dependen del criterio de cada docente a lo hora de evaluar (Facio \& Resett, 2012; Facio et al., 2006).

En lo referente a la relación entre ambas variables, se ha comprobado que los puntajes de atención son un predictor del rendimiento escolar, porque los alumnos con las mejores calificaciones o puntuaciones en los tests estandarizados son -generalmente- quienes mostraban puntajes más elevados en atención selectiva, mejor rendimiento en atención dividida y cometen menos errores en las pruebas de desempeño de atención.

En este sentido, muchas investigaciones demostraron que los alumnos inquietos, con dificultad para permanecer sentados en el aula, distraídos, con altos niveles de impulsividad y con peores puntuaciones en las pruebas de atención, obtenían resultados escolares más bajos -medida a partir de las calificaciones escolares- (Boujon \& Quaireau, 1999; Bresleau et al., 2009). Por ejemplo, un estudio con 600 adolescentes de una muestra comunitaria detectó que un bajo rendimiento en las pruebas de atención a 
los 6-11 años se asociaba a los 11-17 años con un menor rendimiento en Matemática y Lectura (Bresleau et al., 2009).

Del mismo modo, una investigación a partir de seis estudios longitudinales en los Estados Unidos -dos de ellos emplearon muestras representativas de los niños de dicho país- demostraron la relación entre las dificultades de atención en la niñez temprana y el rendimiento escolar (Duncan et al., 2007). Dicho estudio comprobó que las competencias en atención -pero no las competencias sociales y emocionales- en la niñez temprana se asociaban con el rendimiento en Lengua y Matemática en la niñez escolar.

Asimismo, los alumnos con déficits atencionales, como trastorno por déficit de atención, presentan problemas en el aprendizaje de la lectura o de la escritura (Breslau et al., 2009; García-Ogueda, 2001; González-Castro et al., 2008). A este respecto, una investigación comprobó que los niños que asistían a la educación primaria con problemas atencionales -trastorno por déficit de atención- presentaban un peor rendimiento escolar (Rabiner et al., 2016). Está bien documentado que los niños y los adolescentes diagnosticados con dicho trastorno presentan mayor probabilidad de tener peores calificaciones, de repetir grados o de lograr menos años de escolaridad (Baweja et al., 2015).

No obstante, las funciones cognitivas son una de las tantas variables que predicen el rendimiento escolar. En este sentido, la investigación científica señala que otras variables también son importantes a este respecto: el nivel socioeconómico, el nivel de motivación de los alumnos, el ambiente escolar, la involucración parental, entre otros (Boonk et al., 2018; Sirin, 2005; Steinberg, 1997).

\section{El presente estudio}

En la Argentina, como en otras naciones de la América Latina, no existen muchas investigaciones que hayan evaluado la relación entre la atención y las calificaciones escolares. A pesar de que algunos estudios documentaron el bajo rendimiento escolar de los niños y los adolescentes de dicho país, no trataron de determinar si la atención era un predictor significativo (Facio et al., 2006; Facio \& Resett, 2012). Sin embargo, no existen estudios en la Argentina que hayan examinado si el desempeño atencional es un predictor del rendimiento escolar para niños y adolescentes. Al respecto, la mayoría de las investigaciones examinó si existía una relación entre ambas -generalmente, al evaluar síntomas de trastorno por déficit de atención en niños (por ejemplo, Scandar, 2013)-, pero no evaluaron si la atención era un predictor significativo en niños y adolescentes de población comunitaria con un test de reconocidas propiedades psicométricas.

En el Programa Internacional para la Evaluación Educativa (PISA), el cual mide las competencias en Matemática, Lengua y Ciencias, la Argentina se ubica 52 en lectura, por debajo de otros países de la región como Chile, Uruguay y México (Organización para la Cooperación y el Desarrollo Económico [OCDE], 2006), lo que sugiere un bajo nivel de competencias escolares. De este modo, el poder determinar si el desempeño atencional es un predictor del rendimiento escolar puede servir para que futuros estudios traten de incrementar los procesos atencionales con el fin de favorecer un mejor rendimiento académico en niños y adolescentes de población comunitaria. 


\section{Objetivo general}

-Examinar la relación entre la atención y las calificaciones escolares en niños y adolescentes.

\section{Objetivos específicos}

-Evaluar la atención y las calificaciones escolares de los niños y los adolescentes.

-Examinar si niños y adolescentes difieren en el desempeño atencional.

-Determinar si la atención es un predictor de las calificaciones escolares en niños y adolescentes.

\section{Hipótesis}

-Los adolescentes, en comparación con los niños, muestran un desempeño atencional más alto.

-La atención es un predictor significativo de las calificaciones escolares en niños y adolescentes.

\section{Método}

\section{Diseño}

Se trató de un estudio de tipo cuantitativo de tipo descriptivo-correlacional, con un diseño transversal, según el tiempo.

\section{Muestra}

Se constituyó una muestra no probabilística intencional de tres cursos de alumnos de escuela primaria privada en Paraná, provincia de Entre Ríos, Argentina, de 73 alumnos de $4 .^{\circ}$ grado (dos cursos) y $5 .^{\circ}$ grado (un curso) de escuela privada ( $50 \%$ mujeres, edad media $=9.02$, con edades entre 9 y 10 años). También, se constituyó una muestra intencional de 82 alumnos de dos cursos de $3 .^{\circ}$ año y $4 .^{\circ}$ año de educación secundaria, que asistían a una escuela privada en Paraná, Entre Ríos, Argentina (51\% mujeres, edad media $=14.2$, con edades entre 14 y 15 años). Se escogieron dichas edades en los niños por razones de factibilidad -trabajar con niños más pequeños; por ejemplo, 6 o 7 años, hubiese demandado mayor cantidad de tiempo en la implementación del test -y por razones evolutivas- a dicha edad deben haber logrado leer con fluidez y haber consolidado la conciencia fonológica (Aguilar et al., 2011; Arancibia et al., 2012). En el caso de los adolescentes, se escogió la etapa de la adolescencia media (Griffa \& Moreno, 2008) debido a que dicha subetapa presenta las características típicas. Además, las edades de 14 y 15 años permiten una mejor comparación evolutiva con la niñez, porque adolescentes de 12 o 13 años recién están entrando en los cambios puberales de este período. Los criterios de inclusión para el grupo de niños eran tener cumplidos 9 o 10 años, asistir a $4 .^{\circ}$ grado o $5 .^{\circ}$ del sistema educativo primario de la escuela argentina, ser alumno de escuela privada, tener el consentimiento informado por escrito de los padres o tutores legales y no tener problemas visuales o de percepción -que impide realizar el test de atención correctamente. Además, el test de atención no puede aplicarse antes de los 8 años de edad. Con respecto a los adolescentes, los criterios de inclusión eran tener 14 o 15 años, ir a $3 .^{\circ}$ año o $4 .^{\circ}$ del sistema educativo secundario de la escuela argentina, asistir a escuela privada, tener el consentimiento informado por escrito de los progenitores o tutores legales y no tener problemas visuales. Solamente, nueve sujetos 
RELACIÓN ENTRE LA ATENCIÓN Y EL RENDIMIENTO ESCOLAR EN NIÑOS Y ADOLESCENTES

fueron excluidos; ocho por no contar con autorización parental y uno por presentar problemas visuales. Por motivos de confidencialidad, no se brindan los nombres de las escuelas evaluadas.

\section{Instrumentos}

d2 Test de Atención de Brickenkamp (2002) fue usado para evaluar el desempeño atencional en los niños y adolescentes de la presente muestra. El d2 es un test de papel y lápiz que consiste en procesar o trabajar a partir de 14 renglones con 47 caracteres cada uno. Estos caracteres o estímulos contienen las letras "d" o "p", los cuales pueden estar acompañados de una o dos pequeñas rayitas o guiones situados individualmente o en pareja ubicados en la parte superior o inferior del renglón. La consigna, que se les da a los sujetos, es revisar atentamente -de izquierda a derecha- el contenido de cada línea y marcar o hacer un círculo en la "d" que contenga las dos rayitas. Los puntajes fundamentales de este instrumento son: $\mathrm{TR}=$ total de respuestas (número de elementos intentados o procesados); $\mathrm{TA}=$ total de aciertos (número de elementos correctos o acertados) y el TOT que es el número total de elementos procesados menos errores u omisiones. En adelante, dichos indicadores se referenciarán como TR, TA y TOT. El instrumento brinda una medida del control atencional e inhibitorio, por un lado, y de la relación entre velocidad y precisión, por el otro lado. Se puede aplicar desde los 8 años de edad en adelante, por lo cual puede ser usado en niños mayores, adolescentes y adultos. Se puede tomar en forma individual o colectiva. Generalmente, lleva alrededor de 8-10 minutos para ser administrado. Es una de las mediciones de atención más usadas en la literatura mundial y los estudios a este respecto demostraron que presenta buenas propiedades psicométricas, como validez de constructo con la impulsividad (Lozano et al., 2015), confiabilidad por encima de .80 y confiabilidad test/re test con .93 (Brickenkamp, 2002; Steinborn et al, 2017). Fue usado en otros estudios de la Argentina con adecuadas propiedades psicométricas (Filippetti \& Richaud de Minzi, 2012; Muchiut, 2013). Como se señaló, la atención es un proceso encargado de seleccionar los estímulos relevantes del contexto (Pashler, 1998), por lo cual, esta prueba se adapta a dicha definición debido a que mide la atención selectiva como la velocidad de procesamiento (Jiménez et al., 2012). En el presente estudio se usaron solamente el TR y TA, porque se encuentran entre los indicadores más relevantes y fiables (Pawlowski, 2020); además, los otros indicadores se usaron para otras investigaciones. El índice de alfa de Cronbach en la presente investigación fue de .83 para los niños y de .81 para los adolescentes.

Rendimiento escolar. Para evaluarlo se solicitaron a los directivos las calificaciones de todas las materias correspondientes al último trimestre que realiza la institución: Matemática, Lengua, Catequesis, Educación Física, Música, Artes Plásticas, Ciencias Sociales y Ciencias Básicas en los niños -materias obligatorias para todos- y Matemática, Lengua y Literatura, Formación Ética y Ciudadana, Educación Física, Música, Artes Plásticas, Inglés, Historia, Geografía y Biología para los adolescentes -las cuales son obligatorias en el sistema educativo argentino. Las calificaciones se consideran un indicador del rendimiento, tal como se indicó (Galicia-Moyeda et al., 2013; Pizarro et al., 1987).

Cuestionario ad hoc: en el cual se preguntó edad, género, entre otros.

\section{Procedimientos de recolección de datos}

En primer lugar, se contactó a los directivos de las escuelas para explicar el fin del estudio y solicitar su autorización. Luego, se contactó a los progenitores o tutores legales de los niños y adolescentes 
para pedir su consentimiento por escrito mediante una nota en el cuaderno de comunicaciones. El cuaderno de comunicaciones es el instrumento de comunicación formal y obligatoria, que permite la comunicación entre la escuela y los progenitores o tutores legales de los estudiantes. Se aseguró el anonimato, la confidencialidad y la participación voluntaria. Los instrumentos de recolección de datos se aplicaron en el horario de clases en forma grupal y se tardó alrededor de 15 minutos en ser completados. Los instrumentos se aplicaron en las horas libres o durante los períodos de clase que la escuela destinó para este fin. Los autores del manuscrito aplicaron el test en compañía de un docente del establecimiento.

\section{Análisis estadístico}

Los datos se analizaron en el Programa Estadístico para las Ciencias Sociales SPSS 23 para extraer estadísticos descriptivos (porcentajes, medias, entre otros) e inferenciales (regresiones lineales múltiples y comparaciones de medias). Para el caso de las regresiones lineales múltiples, los puntajes en el d 2 se colocaron como predictores y las calificaciones como variables dependientes. Para las comparaciones de medias (con pruebas $t$ de student por el tamaño pequeño de la muestra), los puntajes del test de atención se colocaron como variables dependientes, mientras que la pertenencia a los grupos (ser niño o adolescente) se introdujo como factor entre sujetos. Al igual que otros estudios, se usó el procesamiento de datos paramétricos para los resultados del d2 (por ejemplo, Jiménez et al., 2012) y para las calificaciones (por ejemplo, Galicia-Moyeda et al., 2013). Además, su distribución era relativamente normal con respecto a los valores de curtosis y asimetría, tanto en los niños como los adolescentes. Para los niños, los valores de asimetría en cuanto al total de respuestas y aciertos oscilaban entre .66 a 1.42, mientras que los de curtosis de .07 a 3.07. Para el grupo de adolescentes, fluctuaban entre .50 a 1.79 y entre .04 a 3.98 , respectivamente. En el caso de los niños, los valores de asimetría y curtosis para las calificaciones escolares se registraron entre .87 y 1.38 , y entre .05 y 2.90 , respectivamente, mientras que en el caso de los adolescentes se registraon entre .72 y 1.26 , y entre .08 y 2.44 , respectivamente. Estos índices estaban cercanos a la normalidad, porque valores de asimetría mayores a 3 y de curtosis de 8 a 20 o más son considerados extremos (Kline, 2015); por lo tanto, se usaron pruebas paramétricas.

Teóricamente, existe una asociación lineal entre las variables, lo cual es otro requisito para procesar este tipo de estadísticos (Tabachnick \& Fidell, 2013). Antes de llevar a cabo las regresiones lineales múltiples, se exploraron las relaciones entre las variables con $r$ de Pearson. Se escogió un nivel de significación de $p<.05$, que corresponde al típicamente empleado en las Ciencias Sociales.

\section{Resultados}

En lo referente a la atención, en la tabla 1 se muestran los puntajes de TR y TA para niños y adolescentes. Los resultados indicaban diferencias estadísticamente significativas en el TR entre los niños y los adolescentes $t=3.71, p<.001$ y para el TA $t=2.34, p<.001$ debido a puntajes más elevados de los adolescentes. 
RELACIÓN ENTRE LA ATENCIÓN Y EL RENDIMIENTO ESCOLAR EN NIÑOS Y ADOLESCENTES

\section{Tabla 1}

Estadísticos descriptivos de TR y TA en el test de atención d2 en niños ${ }^{\mathrm{a}} y$ adolescentes $^{\mathrm{b}}$

\begin{tabular}{lcccccc}
\hline Puntajes & Niños & Min. & Max. & Adolescentes & Min. & Máx. \\
\hline TR & 274.34 & 22.54 & 299.07 & 347.70 & 54.80 & 359.01 \\
& $(54.43)$ & & & $(67.01)$ & & \\
TA & 101.11 & 21.02 & 139.56 & 158.12 & 38.60 & 166.53 \\
& $(33.21)$ & & & $(49.87)$ & & \\
\hline
\end{tabular}

Nota $. \mathrm{TA}=$ total de aciertos (número de elementos correctos o acertados) y el TR $(\mathrm{O}+\mathrm{C})=$ número total de elementos procesados menos errores u omisiones.

${ }^{\mathrm{a}} n=73 .{ }^{\mathrm{b}} n=82$.

En la tabla 2 y 3, se muestran las calificaciones para niños y adolescentes. Las calificaciones más bajas se hallaban en Lengua y las más altas en Catequesis, en el caso de los niños. En cuanto a los adolescentes, las más bajas se encontraban en Lengua y Literatura, mientras que las más elevadas se hallaban en Música.

\section{Tabla 2}

Medias y desviaciones estándar de calificaciones en las distintas materias en niños ${ }^{\mathrm{a}}$

\begin{tabular}{lcc}
\hline Calificaciones & $\mathrm{M}$ & $\mathrm{DE}$ \\
\hline Matemática & 8.09 & 1.14 \\
Lengua & 7.99 & 1.76 \\
Catequesis & 8.96 & 1.44 \\
Ed. Física & 8.30 & 1.05 \\
Música & 8.95 & 1.68 \\
Artes Plásticas & 8.30 & 1.21 \\
Ciencias Sociales & 8.32 & 1.09 \\
Ciencias Básicas & 8.01 & 1.76 \\
\hline
\end{tabular}
${ }^{\mathrm{a}} n=73$. 


\section{Tabla 3}

Medias y desviaciones estándar de calificaciones en las distintas materias en adolescentes ${ }^{\mathrm{a}}$

\begin{tabular}{lcc}
\hline Calificaciones & M & DE \\
\hline Matemática & 7.65 & 1.52 \\
Lengua y Literatura & 7.50 & 1.12 \\
Form. Ética y Ciudadana & 7.76 & 1.01 \\
Ed. Física & 8.44 & 1.87 \\
Música & 8.65 & 1.87 \\
Artes Plásticas & 8.09 & 1.55 \\
Inglés & 7.43 & 1.23 \\
Historia & 8.10 & 1.06 \\
Geografía & 7.73 & 1.32 \\
Biología & 7.89 & 1.87 \\
\hline
\end{tabular}

${ }^{\mathrm{a}} n=82$.

Previamente a realizar las regresiones lineales múltiples, se establecieron correlaciones de Pearson entre los puntajes del d2 y las calificaciones escolares para los niños y adolescentes. Para el grupo de niños, se hallaron correlaciones significativas para las calificaciones y TR y TA $(r=.24$ y .67, $p<.05)$. En el caso de los adolescentes, también se detectaron asociaciones significativas $(r=.29$ y .69, $p<.04)$. Con respecto al resultados de las regresiones lineales múltiples, en el caso de los niños, se observó que el TR y TA predecían las calificaciones en Matemática, Lengua y Ciencias Básicas. Como se muestra en la tabla 4, se explicaba una varianza del 6\%, 4\% y 5\%, respectivamente. Dichas ecuaciones de predicción eran significativas $p<.01$. En el caso de los adolescentes, el TR y TA predecían las calificaciones de Matemática, Lengua, Inglés y Biología. Como se muestra en la tabla 5, se explicaba una varianza del 6\%, $7 \%, 3 \%$ y $5 \%$, respectivamente. Dichas ecuaciones de predicción eran significativas $p<.01$. 
RELACIÓN ENTRE LA ATENCIÓN Y EL RENDIMIENTO ESCOLAR EN NIÑOS Y ADOLESCENTES

\section{Tabla 4}

Predicción de los puntajes de las distintas calificaciones a partir del total de respuestas y total de aciertos en el test de atención d2 en niños ${ }^{\mathrm{a}}$

\begin{tabular}{|c|c|c|c|c|c|}
\hline Materias & $\mathrm{R}^{2}$ & Predictor & $\beta$ & $t$ & $p$ \\
\hline \multirow[t]{2}{*}{ Matemática } & $6 \%$ & TR & .118 & 3.073 & .020 \\
\hline & & TA & .211 & 4.332 & .001 \\
\hline \multirow[t]{2}{*}{ Lengua } & $4 \%$ & TR & .140 & 2.275 & .023 \\
\hline & & TA & .160 & 2.294 & .021 \\
\hline \multirow[t]{2}{*}{ Catequesis } & - & TR & .020 & .042 & .622 \\
\hline & & TA & .032 & .057 & .559 \\
\hline \multirow[t]{2}{*}{ Ed. Física } & - & TR & .012 & .035 & .669 \\
\hline & & TA & .011 & .033 & .682 \\
\hline \multirow[t]{2}{*}{ Música } & - & TR & .016 & .039 & .700 \\
\hline & & TA & .011 & .011 & .846 \\
\hline \multirow[t]{2}{*}{ Artes Plást. } & - & $\mathrm{TR}$ & .016 & .023 & 699 \\
\hline & & TA & .009 & .010 & .866 \\
\hline \multirow[t]{2}{*}{ Ciencias Soc. } & & TR & .056 & 1.233 & .211 \\
\hline & & TA & .055 & 1.220 & .243 \\
\hline \multirow[t]{2}{*}{ Ciencias Bás. } & $5 \%$ & TR & .133 & 2.117 & .031 \\
\hline & & TA & .134 & 2.120 & .030 \\
\hline
\end{tabular}

Nota. $\mathrm{TA}=$ total de aciertos (número de elementos correctos o acertados) y el TR $(\mathrm{O}+\mathrm{C})=$ número total de elementos procesados menos errores u omisiones.

${ }^{\mathrm{a}} n=73$. 
Tabla 5

Predicción de los puntajes de las distintas calificaciones a partir del total de respuestas y total de aciertos en el test de atención d2 en adolescentes ${ }^{\mathrm{a}}$

\begin{tabular}{|c|c|c|c|c|c|}
\hline Materias & R2 & Predictor & $\beta$ & $t$ & $p$ \\
\hline \multirow[t]{2}{*}{ Matemática } & $6 \%$ & TR & .233 & 3.707 & .001 \\
\hline & & TA & .119 & 2.860 & .004 \\
\hline \multirow[t]{2}{*}{ Lengua y Literatura } & $7 \%$ & TR & .101 & 2.297 & .005 \\
\hline & & TA & .111 & 2.773 & .004 \\
\hline \multirow[t]{2}{*}{ For. Ética y Ciudadana } & - & TR & .022 & .044 & .624 \\
\hline & & $\mathrm{TA}$ & .008 & .052 & .923 \\
\hline \multirow[t]{2}{*}{ Ed. Física } & - & TR & .012 & .033 & .685 \\
\hline & & TA & .010 & .029 & .777 \\
\hline \multirow[t]{2}{*}{ Música } & - & TR & .014 & .036 & .712 \\
\hline & & TA & .011 & .022 & .824 \\
\hline \multirow[t]{2}{*}{ Artes Plásticas } & - & TR & .010 & .027 & .756 \\
\hline & & TA & .008 & .021 & .788 \\
\hline \multirow[t]{2}{*}{ Inglés } & $3 \%$ & TR & .090 & 2.064 & .041 \\
\hline & & $\mathrm{TA}$ & .010 & 2.088 & .040 \\
\hline \multirow[t]{2}{*}{ Historia } & - & TR & .014 & .036 & .712 \\
\hline & & TA & .013 & .033 & .789 \\
\hline \multirow[t]{2}{*}{ Geografía } & - & TR & .050 & .922 & .354 \\
\hline & & TA & .056 & .622 & .669 \\
\hline \multirow[t]{2}{*}{ Biología } & $5 \%$ & TR & .015 & 2.822 & .005 \\
\hline & & $\mathrm{TA}$ & .017 & 2.873 & .005 \\
\hline
\end{tabular}

${ }^{\mathrm{a}} n=82$.

\section{Discusión}

El propósito de la presente investigación era determinar si los puntajes de la atención eran un predictor significativo del rendimiento escolar en niños y adolescentes y si existían diferencias en el desempeño atencional entre ambos grupos. Para este fin, se constituyó una muestra intencional de alumnos de escuela primaria privada en Paraná, Entre Ríos, Argentina. Así, se conformó una muestra de 73 alumnos de tres cursos de $4 .^{\circ}$ grado y $5 .^{\circ}$ grado de escuela privada y una de 82 alumnos de dos cursos de $3 .^{\circ}$ año y $4 .^{\circ}$ de educación secundaria que asistían a una escuela privada en Paraná, Entre Ríos, Argentina. Con el fin de evaluar la atención, se empleó el D2 Test de Atención de Brickenkamp (2002) y el rendimiento escolar se midió con las calificaciones de todas las materias solicitadas a la escuela. 
RELACIÓN ENTRE LA ATENCIÓN Y EL RENDIMIENTO ESCOLAR EN NIÑOS Y ADOLESCENTES

En lo relativo a explorar el desempeño en atención, se halló un total de respuestas de 274.34 y un total de acierto de 101.11 en los niños, mientras que para los adolescentes los puntajes fueron 347.70 y 158.12, respectivamente. Dichas diferencias entre niños y adolescentes eran estadísticamente significativas. Por ende, se cumple y se acepta la hipótesis que afirmaba que el desempeño atencional de los adolescentes, en comparación con los niños, sería más elevado. Se ha sugerido que un incremento en el desempeño atencional se haya sujeto a la maduración de diferentes estructuras cerebrales, también los cambios o las diferencias aquí detectados pueden deberse a otros factores, tales como el contexto social y cultural; por ejemplo, que se trata de dos cohortes diferentes. El tipo de estudio transversal no permite inferir a cuáles factores se deben estas diferencias. Al comparar estos resultados con los de otros estudios extranjeros, otras investigaciones internacionales hallaron 317.77 de total de respuestas y 127.87 de aciertos en niños de la misma edad. De este modo, los resultados en la muestra de niños, aquí examinada, mostraban un rendimiento inferior al de otras investigaciones (Jiménez et al., 2012). Se debe aclarar que los resultados de dicha investigación eran de una muestra española; por lo tanto, las diferencias podrían deberse a factores sociales, culturales y económicos entre ambos países. Un estudio en la Argentina con niños de la misma edad evidenció valores similares con un total de respuestas de 282.79 y total de aciertos de 109.49 (Muchiut, 2013). No se han detectado estudios en adolescentes argentinos para comparar los presentes resultados. Sin embargo, el tipo de muestra intencional no permite generalizarlos.

Con respecto a las calificaciones, en los niños se hallaban entre 8 y 9 -en la Argentina las calificaciones van de 1 a 5, lo cual implica que el sujeto no aprueba los contenidos; entre 6 a 7 es aprobado; entre 8 a 9, un muy buen desempeño y un 10, desempeño excelente. Las calificaciones más altas se presentaban en Música y Catequesis; las más bajas eran en Matemática, Lengua y Ciencias Básicas. En los adolescentes, las calificaciones fluctuaban entre el 7.50 y 8.50, con los valores más elevados en Música -también- y educación física, mientras que las puntuaciones más bajas eran en Lengua y Literatura, Inglés y Matemática. Habría que evaluar por qué el rendimiento más elevado se haya en las materias artísticas como música en ambos casos: ¿depende de las exigencias del docente?, ¿niños y adolescentes se sienten más motivados hacia este tipo de disciplina? o ¿se debe a una cuestión del contenido disciplinar mismo? Que las calificaciones más bajas se observen en materias como Matemática o Lengua no es sorprendente. Por ejemplo, en las pruebas internacionales del Progress in International Reading Literacy (PIRL, 2001), los niños de cuarto grado de la Argentina se ubicaban en los últimos puestos y eran superados por otros países de la región; por ejemplo, Colombia. Asimismo, estudios internacionales con adolescentes han demostrado que el rendimiento escolar de los adolescentes argentinos es mucho más bajo que el de otros países de la región, tal como lo indican los estudios del PISA (OCDE, 2012). Esto mismo se ha encontrado en Matemática y ciencia con los niños argentinos de cuarto grado, como lo indican los estudios del Trend in International Mathematics and Science Study (TIMSS, 2015). Asimismo, dicho estudio demuestra un deterioro progresivo en el rendimiento en dichas materias con el paso de los años. También, investigaciones nacionales comprobaron que el rendimiento escolar de niños y adolescentes en la Argentina es inferior en materias como Matemática y Lengua, incluso ellos mismos indicaban que su gran fracaso son las tareas escolares (Facio et al., 2006). Futuras investigaciones deberían examinar a cuáles factores se debe este estado de situación: problemas de formación docente, de falta de recursos en las escuelas o un menor número de días de clases anuales -incluso en comparación con países de la región como Chile o Uruguay- (Facio \& Resett, 2012). Esto es sumamente alarmante, porque está 
establecido que un buen rendimiento académico o escolar es una importante tarea del desarrollo. Así, un rendimiento académico precario es un gran factor de riesgo no solo concurrente, sino también a largo plazo: bajas calificaciones se asocian a futuro con una peor salud mental, trabajos con baja remuneración, entre otros (Steinberg, 2019). No obstante, en el presente estudio, las calificaciones de los niños en Matemática y Lengua demostraban un muy buen rendimiento -a pesar de ser las más bajas- y en los adolescentes eran alrededor de 7.50 -por lo cual, el adolescente promedio aprobaba dicho contenido. Sin embargo, las calificaciones son subjetivas y dependen del criterio del docente a diferencia de las evaluaciones estandarizadas internacionales.

En el caso de los niños, se observó que el total de respuestas y aciertos predecían las notas en Matemática, Lengua y Ciencias Básicas. En el caso de los adolescentes, también el total de respuestas y aciertos predecían las notas de Matemática, Lengua, Inglés y Biología. Estos hallazgos son similares a los de numerosos estudios extranjeros en muestras de niños y adolescentes, los cuales demostraron que un mejor desempeño atencional se asociaba con mayores puntuaciones en las calificaciones, tanto concurrente como a largo plazo (Breslau et al., 2009; Duncan et al., 2007), principalmente en Matemática y Lengua. Estos resultados ponen de relieve que la atención es un componente significativo para el rendimiento escolar, tanto en niños como adolescentes. Por todo lo dicho, se cumple y se acepta la hipótesis que sostenía que el desempeño atencional era un predictor de las calificaciones escolares en niños y adolescentes. Futuros estudios deberían examinar si en Matemática, Lengua y Ciencia -en comparación con las otras disciplinas- es necesario un mayor desempeño atencional o si, en dichas materias, hay una mayor exigencia de parte de los docentes en las evaluaciones. Algunos estudios sugieren que, por ejemplo, la manipulación de información (memoria de trabajo), como la supresión de distractores (inhibición), son centrales en el aprendizaje de las Matemáticas (Cragg \& Gilmore, 2013); a este respecto, es necesaria mayor investigación. Sin embargo, los tamaños del efecto detectados en las calificaciones eran pequeños (no superaban el 8\%); pero, la investigación empírica indica que, en Psicología, los fenómenos están multideterminados. Algunos estudios indicaron que el desempeño académico está no solo determinado por factores cognitivos -como inteligencia o memoria de trabajo-, sino también por factores sociales o emocionales, tales como motivación, estilos de crianza, actitudes de los docentes, estilos de aprendizaje, el valor que la familia le da a la escuela, entre otros (Sirin, 2005; Steinberg, 1997). No obstante, estos resultados son coincidentes con los de Duncan et al. (2007), en los Estados Unidos, quienes detectaron que las competencias cognitivas en niños -no las emocionales ni sociales- predecían el rendimiento escolar. Además, es posible que un conjunto de funciones cognitivas -por ejemplo, funciones ejecutivas, inteligencia fluida, entre otras-, sean las responsables de un mejor rendimiento escolar. Por lo tanto, futuras investigaciones deberían examinar el perfil cognitivo de alumnos más o menos exitosos a nivel escolar.

Esta investigación contiene una serie de limitaciones; en primer lugar, la muestra seleccionada en forma intencional no permite la generalización de los resultados. En segundo lugar, el tamaño de la muestra no posibilita análisis estadísticos más complejos como los modelos estructurales. En tercer lugar, el rendimiento escolar se evaluó con las calificaciones, las cuales son un indicador del rendimiento y dependen del criterio de cada docente (Facio et al., 2006). En cuarto lugar, el test de atención autoadministrado puede haber llevado a sesgos en los resultados o a que los alumnos, principalmente los niños, hayan malinterpretado las consignas. 
RELACIÓN ENTRE LA ATENCIÓN Y EL RENDIMIENTO ESCOLAR EN NIÑOS Y ADOLESCENTES

Futuros estudios deberían ser desarrollados con muestras de mayor tamaño, seleccionadas al azar y de distintas regiones de la Argentina con el fin de generalizar los resultados. Asimismo, se deberían emplear mediciones estandarizadas para el rendimiento escolar con el fin de evitar el sesgo en las calificaciones, como pueden ser las pruebas que emplea el Programa para la Evaluación Internacional de Alumnos (OCDE, 2007) en adolescentes u otras medidas estandarizadas para los niños, como el Test de Lectura y Escritura (Citoler et al., 2007), que es una prueba de lectura y escritura o el test Pro-Calculo (Feld et al., 2006) para las habilidades aritméticas que están estandarizadas al español. También, se deben considerar otros indicadores del rendimiento escolar, como el ausentismo, la actitud hacia la escuela, entre otros. Además, se deberían emplear otras mediciones de atención además del d2, como el STROOP Test de Palabras y Colores (Golden, 2005). Del mismo modo, como el rendimiento escolar es una variable multidimensional, también futuros trabajos deberían medirlo con indicadores múltiples, como los años de escolaridad, la actitud hacia la escuela, el ausentismo, entre otros.

En otro orden, futuros estudios deberían ser longitudinales con el fin de determinar cómo covarían mediante el paso del tiempo, la atención y el rendimiento escolar. Del mismo modo, este tipo de estudio podría determinar si la atención influye en el rendimiento escolar u otras variables neuropsicológicas generan covarianzas -esto es, evaluar la direccionalidad de la causalidad. Finalmente, se deberían incorporar otras variables relevantes para el rendimiento escolar: inteligencia, nivel socioeconómico, motivación, entre otros, así como realizar un perfil cognitivo de los alumnos con mejor y peor desempeño académico.

\section{Referencias}

Aguilar, M., Marchena, E., Navarro, J.I., Menacho, I., \& Alcalde, C. (2011). Niveles de dificultad de la conciencia fonológica y aprendizaje lector. Revista de Logopedia, Foniatría y Audiología, 31, 96-105. https://doi. org/10.1016/S0214-4603(11)70177-2

Arancibia, B., Bizama, M., \& Sáez, K. (2012). Aplicación de un programa de estimulación de la conciencia fonológica en preescolares de nivel transición 2 y alumnos de primer año básico pertenecientes a escuelas vulnerables de la Provincia de Concepción, Chile. Revista Signos. Estudios de Lingüística, 45, 236-256. http://dx.doi.org/10.4067/S0718-09342012000300001

Arnett, J. J. (2012). Adolescencia y adultez emergente. Un enfoque cultural (5a ed.). Pearson.

Asociación Americana de Psiquiatría (2013). Manual Diagnóstico y Estadístico de los Trastornos Mentales (5a ed.). Asociación Americana de Psiquiatría.

Barkley, R.A. (1997). ADHD and the nature of self-control. Guilford Press.

Baweja, R., Mattison, R. E., \& Waxmonsky, J. G. (2015). Impact of Attention-Deficit Hyperactivity Disorder on School Performance: What are the Effects of Medication? Pediatric Drugs, 17(6), 459-477. https://doi.org/10.1007/s40272-015-0144-2 
Biederman, J., Monuteaux, M. C., Doyle, A. E., Seidman, L. J., Wilens, T. E., Ferrero,..., Faraone, S. V. (2004). Impact of Executive Function Deficits and Attention-Deficit/Hyperactivity Disorder (ADHD) on Academic Outcomes in Children. Journal of Consulting and Clinical Psychology, 72(5), 757-766. https://doi. org/10.1037/0022-006X.72.5.757

Boonk, L. Gijselaers, H., Ritzen, H., \& Brand-Gruwel, S. (2018). A review of the relationship between parental involvement indicators and academic achievement. Educational Research Review, 24, 10-30. https://doi. org/10.1016/j.edurev.2018.02.001

Boujon, CH. \& Quaireau, CH. (1999). Atención, aprendizaje y rendimiento escolar. Narcea.

Brefczynski-Lewis, J. A., Lutz, A., Schaefer, H. S., Levinson, D. B., \& Davidson, R. J. (2007). Neural correlates of attentional expertise in long-term meditation practitioners. Proceedings of the National Academy of Sciences U.S.A., 104, 11483-11488. https://doi.org/10.1073/pnas.0606552104

Breslau, J., Miller, E., Breslau, N., Bohnert, K., Lucia, V., \& Schweitzer, J. (2009). The impact of early behavior disturbances on academic achievement in high school. Pediatrics, 123, 1472-1476. https://doi.org/10.1542/ peds.2008-1406

Brickenkamp, R. (2002). d2 Test de atención (N. Seisdedos, Trad.). TEA Ediciones.

Bryck, R. L., \& Fisher, P. A. (2011). Training the Brain: Practical Applications of Neural Plasticity From the Intersection of Cognitive Neuroscience, Developmental Psychology, and Prevention Science. American Psychologist, 67(2), 87-100.

Caso-Niebla, J., \& Hernández-Guzmán, L. (2007). Variables que inciden en el rendimiento académico de adolescentes mexicanos. Revista Latinoamericana de Psicología, 39(3), 487-501

Chun, M., \& Turk-Browne, N. (2007). Interactions between attention and memory. Current Opinion in Neurobiology, 17, 177-184. https://doi.org/10.1016/j.conb.2007.03.005

Citoler, S., Fonseca, L., Gottheil, B., Aldrey, A. Pujals, M., Rosa, G.,..., Serrano-Chica, F. (2007). LEE. Test de Lectura y Escritura en Español. Psicología y Psicopedagogía, 6(1)7, 1-7.

Cragg, L., \& Gilmore, C. (2014). Skills underlying mathematics: The role of executive function in the development of mathematics proficiency. Trends in Neuroscience and Education, 3(2), 63-68. https://doi.org/10.1016/j. tine.2013.12.001

Dosher, B. A., \& Lu, Z. L. (2000). Noise exclusion in spatial attention. Psychological Science, 11(2), 139-146. https://doi.org/10.1111/1467-9280.00229

Duncan G.J., Dowsett C.J., Claessens A., Magnuson K., Huston A.C., Klebanov P., Pagani L.S., Feinstein L., Engel M., Brooks-Gunn J., Sexton H.,... Crista, J. (2007). School readiness and later achievement. Developmental Psychology, 43, 1428-1446. https://doi.org/10.1037/0012-1649.43.6.1428

Facio, A., \& Resett, S. (2012). Argentina. En J. Arnett (Ed.), Adolescent Psychology around the World (pp. 151162). Psychology Press.

Facio, A., Resett, S., Mistrorigo, C., \& Micocci, F. (2006). Adolescentes Argentinos. Editorial Lugar. 


\section{RELACIÓN ENTRE LA ATENCIÓN Y EL RENDIMIENTO ESCOLAR EN NIÑOS Y ADOLESCENTES}

Fan, J., McCandliss, B. D., Fossella, J., Flombaum, J. I., \& Posner, M. I. (2005). The activation of attentional networks. Neuroimage, 26(2), 471-479. https://doi.org/10.1016/j.neuroimage.2005.02.004

Fan, J., McCandliss, B. D., Sommer, T., Raz, A., \& Posner, M. I. (2002). Testing the efficiency and independence of attentional networks. Journal of Cognitive Neuroscience, 14(3), 340-347. https://doi. org/10.1162/089892902317361886

Feld, V., Taussik, I., \& Azaretto, C. (2006). Manual del Pro-Cálculo: test para la evaluación del procesamiento del número y el cálculo en niños. Paidós.

Filippetti, V., \& Richaud de Minzi, M. C. (2012). Análisis de la relación entre reflexividad-impulsividad y funciones ejecutivas en niños escolarizados mediante un modelo de ecuaciones estructurales. International Journal of Psychology and Psychological Therapy, 12(3), 427-440. https://www.redalyc.org/articulo. oa? id $=56024657008$

Galicia-Moyeda, I., Sánchez-Velasco, A., \& Robles-Ojeda, F. (2013). Autoeficacia en escolares adolescentes: su relación con la depresión, el rendimiento académico y las relaciones familiares. Anales de Psicología, 29, (2), 491-500. http://dx.doi.org/10.6018/analesps.29.2.124691

García-Ogueda, M. (2001). Mecanismos atencionales y síndromes neuropsicológicos. Revista de Neurología, $32(5), 463-467$.

Gogtay, N., Giedd, J. N., Lusk, L., Hayashi, K. M., Greenstein, D., Vaituzis, A. C., \&., Thompson, P. M. (2004). Dynamic mapping of human cortical development during childhood through early adulthood. Proceedings of the National Academy of Sciences, USA, 101, 8174-8179. https://doi.org/10.1073/pnas.0402680101

Golden, C. J. (2005). STROOP Test de colores y palabras. TEA ediciones.

González-Castro, P., Álvarez, L., Núñez, J.C., González-Pienda, J.A. \& Álvarez, D. (2008). La aplicación de una estrategia computarizada para enseñar y aprender matemáticas en Educación Primaria. European Journal of Education and Psychology, 1(1), 21-38. https://doi.org/10.30552/ejep.v1i1.3

Gottlieb, J. (2007). From Thought to Action: The Parietal Cortex as a Bridge between Perception, Action, and Cognition. Neuron, 53(1), 9-16. https://doi.org/10.1016/j.neuron.2006.12.009

Griffa, M.C. \& Moreno, J.E. (2008). Claves para una psicología del desarrollo (Vol. 2). Editorial Lugar.

James, W. (1890). The principles of psychology. Dover Publications.

Jiménez, J., Hernández, S., García, E., Díaz, A., Rodríguez, C \& Martín, R. (2012). Test de atención D2: Datos normativos y desarrollo evolutivo de la atención en educación primaria. European Journal of Education and Psychology, 5(1), 93-106. https://doi.org/10.30552/ejep.v5i1.79

Kline, R. (2015). Principles and practice of structural equation modeling (4th ed.). Guilford.

LeFever, G. B., Villers, M. S., Morrow, A. L., \& Vaughn, E. S (2002). Parental perceptions of adverse educational outcomes among children diagnosed and treated for ADHD: a call for improved school/provider collaboration. Psychology in the Schools, 39, 63-71. https://doi.org/10.1002/pits.10000 
Lozano, J. H., Capote, E., \& Fernández, M. P. (2015). Convergent validity of the measures of attention and impulsivity in the Trees: Simple Visual Discrimination Test (DiViSA-UAM). Anales de Psicología, 31(1), 74-83. https://doi.org/10.6018/analesps.31.1.161371

Luck, S. J., Hillyard, S. A., Mouloua, M., \& Hawkins, H. L. (1996). Mechanisms of visualspatial attention: Resource allocation or uncertainty reduction? Journal of Experimental Psychology: Human Perception and Performance, 22(3), 725-737. https://doi.org/10.1037/0096-1523.22.3.725

Mangun, G. R., \& Hillyard, S. A. (1991). Modulations of sensory-evoked brain potentials indicate changes in perceptual processing during visual-spatial priming. Journal of Experimental Psychology: Human Perception and Performance, 17(4), 1057-1074. https://psycnet.apa.org/doi/10.1037/0096-1523.17.4.1057

Manes, F. (2014). Usar el cerebro. Planeta.

Muchiut, A. (2013). El perfil atencional en niños [Manuscrito inédito].

Organización para la Cooperación y el Desarrollo Económico (2006). Programa para la Evaluación Internacional de Alumnos. PISA 2006: Science Competencies for Tomorrow's World. UNESCO.

Organización para la Cooperación y el Desarrollo Económico. (2012). Programa para la Evaluación Internacional de Alumnos. PISA 2012. UNESCO.

Pashler, H. E. (1998). The psychology of attention. The MIT Press.

Pawlowski, J. (2020). Test de Atención d2: Consistencia interna, estabilidad temporal y evidencias de validez. Revista Costarricense de Psicología, 39(2), 145-165. http://dx.doi.org/10.22544/rcps.v39i02.02

Petersen, S. E., \& Posner, M. I. (2012). The attention system of the human brain: 20 years after. Annual Review of Neuroscience, 35, 73-89. https://10.1146/annurev-neuro-062111-150525

Pizarro, R., Clark, L., \& Allen, M. (1987). El ambiente educativo del hogar. Diálogos Educacionales, 9(10), 66-83.

Portellano, J. (2005). Introducción a la neuropsicología. McGraw-Hill.

Posner, M. I., \& Dehaene, S. (1994). Attentional networks. Trends in Neurosciences, 17(2), 75-79. https://doi. org/10.1016/0166-2236(94)90078-7

Posner, M. I., \& Petersen, S. E. (1990). The attention system of the human brain. Annual Review of Neuroscience, $13,25-42$.

Progress in International Reading Literacy Study. (2001). International Comparisons in Fourth-Grade Reading Literacy: Findings from the Progress in International Reading Literacy Study (PIRLS) of 2001. Centro Nacional de Estadísticas.

Rabiner, D., Carrig, M., \& Dodge, K. (2016). Attention problems and academic achievement: Do persistent and earlier-emerging problems have more adverse long-term effects? Journal of Attention Disorder, 20(11), 946-957. https://doi.org/10.1177\%2F1087054713507974

Reynolds, J. H., Pasternak, T., \& Desimone, R. (2000). Attention increases sensitivity of V4 neurons. Neuron, 26(3), 703-714. https://doi.org/10.1016/S0896-6273(00)81206-4 
RELACIÓN ENTRE LA ATENCIÓN Y EL RENDIMIENTO ESCOLAR EN NIÑOS Y ADOLESCENTES

Rios-Lago, M., Muñoz, J.M., \& Paúl, N. (2007). Alteraciones de la atención tras daño cerebral traumático: evaluación y rehabilitación. Revista de Neurología, 44, 291-297. https://doi.org/10.33588/rn.4405.2006208

Rodríguez, J. (2004). La década del cerebro (1990-2000). Revista española de neuropsicología, 6, 131-170.

Rothbart, M. K., Sheese, B. E., Rueda, M. R., \& Posner, M. I. (2011). Developing Mechanisms of Self-Regulation in Early Life. Emotion Review, 3(2), 207-213. https://doi.org/10.1177/1754073910387943

Rumbaugh, D.M., \& Washburn, D.A. (1995). Attention and memory in relation to learning: A comparative adaptation perspective. En G.R. Lyon \& N.A. Krasengor (Eds.), Attention, Memory and Executive Function (pp. 199-219). Brookes Publishing Co.

Rumberger, R. \& Palardy, G. (2005). Test scores, dropout rates, and transfer rates as alternative indicators of high school performance. American Educational Research Journal, 42(1), 3-42. https://doi. org $/ 10.3102 / 00028312042001003$

Scandar, M. (2013). Relación entre los síntomas de TDAH y el aprendizaje escolar en niños preescolares argentinos. Revista Neuropsicología Latinoamericana, 5(2), 11-23. http://dx.doi.org/10.5579/rnl.2013.0139

Sirin, S. R. (2005). Socioeconomic Status and Academic Achievement: A Meta-Analytic Review of Research. Review of Educational Research, 75(3), 417-453. https://doi.org/10.3102/00346543075003417

Slotnick, S. D., Schwarzbach, J., \& Yantis, S. (2003). Attentional inhibition of visual processing in human striate and extrastriate cortex. Neuroimage, 19(4), 1602-1611. https://doi.org/10.1016/S1053-8119(03)00187-3

Steinberg, L. (1997). Beyond the classroom. Simon \& Schuster.

Steinberg, L. (2019). Adolescence (10a. ed.). Mc Graw-Hill.

Steinborn, M., Langner, R., Flehmig, H., \& Huestegge, L. (2017). Methodology of Performance Scoring in the d2 Sustained-Attention Test: Cumulative-Reliability Functions and Practical Guidelines. Psychological Assessment, 30(3), 1-19. https://doi.apa.org/doi/10.1037/pas0000482

Tabachnick, B. G., \& Fidell, L. S. (2013). Using multivariate statistics. Allyn and Bacon/Pearson Education.

Thomas, R., Sanders, S., Doust, J., Beller, E., \& Glasziou, P. (2015). Prevalence of attention-deficit/hyperactivity disorder: a systematic review and meta-analysis. Pediatrics, 135(4), 994-1001. https://doi.org/10.1542/ peds.2014-3482

Trend in International Mathematics and Science Study (2015). TIMSS 2015: International results. International Study Center.

Valle-Arias, A., González-Cabanach, R., Núñez-Pérez, J., Suárez-Riveiro, J., Piñeiro-Aguín, I., \& RodríguezMartínez, S. (2000). Enfoques de aprendizaje en estudiantes universitarios. Psicothema, 12(3), 368-375. http://www.psicothema.com/pdf/344.pdf

Recibido: 16 de mayo de 2018 Revisión recibida: 16 de abril de 2021

Aceptado: 24 de mayo de 2021 


\section{Sobre el autor:}

Santiago Resett es doctor en Psicología y profesor de la Universidad Argentina de la Empresa (Argentina) e investigador del Consejo Nacional de Investigaciones Científicas y Tecnológicas de la Argentina. Se ha especializado en el desarrollo socioemocional de los niños y adolescentes. Labora como docente y tutor en centros y universidades de diferentes países. Se distingue como miembro fundador de la Sociedad para el Estudio de la Adultez Emergente. Cuenta con más de 80 publicaciones, comunicaciones y conferencias en congresos nacionales e internacionales.

Publicado en línea: 30 de junio de 2021 